\title{
How Could We Increase the Impact Factor of the Journal of Neurogastroenterology and Motility?
}

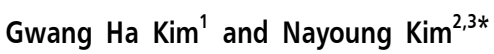 \\ ${ }^{1}$ Department of Internal Medicine, Pusan National University School of Medicine, and Biomedical Research Institute, Pusan National University \\ Hospital, Busan, Korea; ' Department of Internal Medicine, Seoul National University Bundang Hospital, Seongnam, Gyeonggi-do, Korea; and \\ ${ }^{3}$ Department of Internal Medicine and Liver Research Institute, Seoul National University College of Medicine, Seoul, Korea
}

The Journal of Neurogastroenterology and Motility (JNM) is an official journal jointly published by the Korean Society of Neurogastroenterology and Motility, the Thai Neurogastroenterology and Motility Society, the Japanese Society of Neurogastroenterology and Motility, the Indian Motility and Functional Disease Association, the Chinese Society of Gastrointestinal Motility, the South East Asia Gastro-Neuro Motility Association, the Taiwan Neurogastroenterology and Motility Society, and the Asian Neurogastroenterology and Motility Association, that was launched in January 2010. The main aim of JNM is to provide worldwide researchers, especially Asian researchers, an accessible platform for the publication of their research results in the field of neurogastroenterology and motility. Owing to the active participation of members of each society and devotion of the editorial board members, the impact factor (IF) of JNM has been increasing steadily and JNM was finally accepted as one of the Science Citation Index Expanded (SCIE) journals in September, 2013. The IF of JNM in 2013 was 2.698, ranked 31 st among 75 journals in the categories of Gastroenterology and
Hepatology in SCI(E) journals. In addition, the IF of JNM in 2013 was released as 3.537 (18.1 percentile) ranked 24st in the SCOPUS. We expected the IF would increase further. Disappointingly, the self-analyzed IF of JNM in 2014 was found to decrease to 2.194 in the end of March. Of course, it is not final and it could be changed a little until May 2015. However, the decrease is definite and the publication committee of JNM evaluated the possible causes for the decreased IF so that appropriate strategies could be implemented to increase it.

First, the denominator used in calculating the IF had increased. The denominator of IF includes the number of review articles, original articles and case reports. However, we found "How to interpret a functional or motility test" was included in the analysis of 2013 by Thomson Reuters. The number of original articles per issue between 2013 and 2014 was 10 while the number of original articles per issue between 2011 and 2012 was 7. Therefore, it appeared that the increase in number of citations could not catch up with the sudden increase in the number of original articles in recent issues. Second, the Journal contained

Received: March 3, 2015 Revised: March 11, 2015 Accepted: March 12, 2015

(c) This is an Open Access article distributed under the terms of the Creative Commons Attribution Non-Commercial License (http://creativecommons. org/licenses/by-nc/3.0) which permits unrestricted non-commercial use, distribution, and reproduction in any medium, provided the original work is properly cited.

*Correspondence: Nayoung Kim, MD, PhD

Department of Internal Medicine, Seoul National University Bundang Hospital, 173-82, Gumi-ro, Bundang-gu, Seongnam, Gyeonggi-do 463-707, Korea

Financial support: None.

Tel: +82-31-787-7008, Fax: +82-31-787-4051, E-mail: nayoungkim49@empas.com

Conflicts of interest: None.

Author contributions: Gwang Ha Kim wrote this editorial and Nayoung Kim advised the strategies and supervised the editing draft.

ORCID: Nayoung Kim, http://orcid.org/0000-0002-9397-0406. 
poorly cited sections. The frequency of citations for sections such as "Case report" and "How to interpret a functional or motility test" was very low (less than 1). Third, the frequency of citations for recently published review articles decreased, mainly because of the exhaustion of influential review article subjects and authors. Fourth, self-citation of papers published in JNM was not satisfactory. In fact, the self-citation IF in JNM also decreased from 0.417 in 2013 to 0.233 in 2014 . It could be due to the authors of published papers in 2014 did not know much about the JNM papers in 2012 and 2013. It might mean that significant portion of new authors increased who were not familiar with JNM papers in the world. Finally, there could be a tendency to accept relatively low quality or less interesting papers. There are several reasons behind this; sudden increase in the number of submitted papers, inadequate filtration of these papers due to limited number of reviewers, especially in the basic science field, generosity towards papers from Asian countries, and increased acceptance of out of mode "Me too" papers and papers with less interesting subjects.

Then, even though the IF itself is not absolute for evaluating the importance of journals, are there any methods to increase the IF of JNM? The publication committee of JNM had extensive discussions about this issue and came up with the following strategies to increase the IF. First, slimming down of the JNM is essential. To this end, removal of poorly-cited sections such as "Case report" and "How to interpret a functional or motility test", consideration of decreasing the number of published original articles (to 8 ) and review articles (to 2-4), and proper use of early on-line publications are required. Second, the review system for submitted manuscripts should be improved. Allocating associate editors to more specialized fields efficiently, maintaining quality and duty of editorial board members, creating a pool of specialized reviewers according to the subjects in order to decrease reviewers' burden, rewarding high-quality reviewers appropriately, or creating a link to JNM papers similar to the submitted manuscript in the review system can be considered. Third, highly citable subjects are required. To achieve this, it is necessary to ask experienced authors to write review articles as well as original articles. It is also important to find new pioneering authors, especially young investigators, to be able to publish good papers consistently, and potential subjects after analyzing the highly citable papers. Furthermore, there is a tendency that meeting-related publications are poorly citable and that a clinical study is more likely to be cited when compared to a basic study in case of original article. Finally, a few other methods to increase the IF are as follows; to contact authors having highly cited papers consistently, to provide feedback to authors about citation results, or to release e-pub as soon as possible for the most accepted articles.

As stated before, the IF is important to gauge the influence of journals. However, before applying the above-mentioned strategies to increase the IF of JNM, there are some important points to consider. First, this situation could be a transient phenomenon occurring with most journals after being indexed as SCIE. If so, then the IF would recover to the previous level or even surpass it gradually as time goes by. Second, the balance between clinical studies and basic studies and between major subjects and minor subjects is necessary in JNM irrespective of IF. Finally, the aims of JNM such as providing researchers, who cannot have a chance to publish their results due to limited space of journals on the field of neurogastroenterology and motility, with a platform for publication of their results and increasing the base of investigators engaged in this field should be preferentially considered. 